(Respond: Dr Scheffer, Department of Neurology, Austin Hospital, Heidelberg (Melbourne), Victoria, Australia).

COMMENT. The authors suggest that this new syndrome, with its known genetic basis, may help to clarify the relationship between benign rolandic epilepsy, a benign syndrome, and Landau-Kleffner and CSWSS, more severe syndromes.

Symptoms and findings in the Landau-Kleffner (LKS) and continuous spike-and-wave during slow sleep (CSWSS) syndromes are compared in a report from the Department of Child Neurology, University of Helsinki, Children's Castle Hospital, Helsinki, Finland.

(Granstrom M-L et al. Epilepsia 1995;36(suppl 4):123). Bilateral epileptiform activity was found in sleep EEGs in 5 of 6 LKS children and in all 11 children with CSWSS. All LKS children had auditory agnosia and deterioration of expressive language, 4 had attention deficit disorders, and 2 became clumsy or ataxic. Six children with CSWSS had deterioration of expressive language, motor skills and general intelligence, and 4 had hyperkinesia and delayed development. Epileptic seizures occurred in all LKS and in 8 CSWSS children. Mean age at diagnosis was 5 years for LKS and 6 and $1 / 2$ years for CSWSS. MRI/CT was abnormal in 1 LKS and 5 CSWSS patients. LKS usually affects previously normal children whereas CSWSS occurs in children with pre- or perinatal pathology and previously abnormal development. An overnight EEG is recommended in children with developmental arrest, loss of speech, and/or major behavioral problems. Four additional papers on Landau-Kleffner syndrome will be presented at the Annual Meeting of the American Epilepsy Society, Baltimore, Dec 1-6, 1995.

\title{
VALPROATE, BRAIN ATROPHY AND REVERSIBLE DEMENTIA
}

Two children who developed severe cognitive and behavioral deterioration while being treated with sodium valproate for idiopathic epilepsy are reported from the Miami Children's Hospital, Miami, FL. Patient 1 presented at age 5 years with left focal seizures and right central sharp waves on the EEG, consistent with benign rolandic epilepsy (BRE). After his fourth seizure at age 8 years he received sodium valproate (Depakote), with blood levels of 91-106 mg/dl, and methylphenidate (MPH) $20 \mathrm{mg}$ twice daily for an associated ADDH. MPH was replaced by thioridazine (Mellaril) and benztropine (Cogentin) at age 9 years. Impaired motor ability was noted at age 10 years. Activity level, speech, and IQ progressively deteriorated, with ataxia and marked obesity developing by 10 years 8 months. MRI showed enlarged ventricles and cortical sulci. Felbamate was substituted for valproate and his motor activity, speech, gait, weight, and IQ returned to normal within a few weeks. The MRI improved after 3 months and was normal at 1 year. Pemoline (Cylert) was introduced for $\mathrm{ADDH}$ without relapse of behavior or seizure recurrence. Patient 2 had an onset of a similar degenerative syndrome within 3 weeks of prescribing valproate for migraine and BRE. The condition resolved 4 to 6 months after substituting phenobarbital. No metabolic changes were uncovered. (Papazian O et al. Reversible dementia and apparent brain atrophy during valproate therapy. Ann Neurol October 1995;38:687-691). (Respond: Dr Papazian, 3200 SW 60th Ct, \#302, Miami, FL 33155).

COMMENT. Patients treated with valproate need to be monitored for mental and motor deterioration in addition to liver dysfunction. 